PROCEEDINGS OF THE

AMERICAN MATHEMATICAL SOCIETY

Volume 130, Number 10, Pages 2811-2814

S 0002-9939(02)06196-8

Article electronically published on May 8, 2002

\title{
DIRECTED INVERSE LIMITS OF SPATIAL LOCALES
}

\author{
WEI HE AND TILL PLEWE
}

(Communicated by Alan Dow)

\begin{abstract}
In this note we consider spatiality of directed inverse limits of spatial locales. We give an example which shows that directed inverse limits of compact spatial locales are not necessarily spatial. This answers a question posed by John Isbell. We also give a condition which, if satisfied by the maps of a directed inverse system, implies that taking limits preserves local compactness and hence produces spatial locales.
\end{abstract}

The question "Which limits are preserved by the embedding of sober topological spaces into the category of locales?", or equivalently "When is a limit of spatial locales spatial?" seems to be rather difficult. Results concerning preservation of products (and some applications) can be found in [2] 7, 8]. Here we consider preservation of directed inverse limits; in particular Isbell's question whether a directed inverse limit of (quasi-)compact spatial locales is again spatial [1, p. 24]. It is easy to see that if all locales are Hausdorff (i.e. if they have closed diagonals), then this question has a positive answer because (i) compact Hausdorff locales are spatial and closed under products, (ii) limits can be constructed as equalizers of a pair of maps between products of the original objects, and (iii) equalizers of maps between Hausdorff locales are closed inclusions and compactness is inherited by closed sublocales. So only the case of non-Hausdorff locales is problematic.

This note consists of two parts. In the first part we give a construction which shows that directed inverse limits of compact locales need not be spatial even if all locales are furthermore locally compact and all maps open. However, in the second part we show that if all locales are locally compact and all maps proper (a weaker property suffices), then the inverse limit is again locally compact and hence spatial; here compactness is not needed.

Constructions of the limit of a directed inverse system $\left\{X_{i}, f_{j k}: X_{j} \rightarrow X_{k} \mid\right.$ $i, j, k \in I, j \leq k\}$ of locales can be found in [3], 6] or [10]. We don't need to go into any detail here since we will only be using the fact that if $\left\{B_{j}\right\}$ is a collection of bases for the locales $X_{j}$, then the union of the inverse images under the maps $f_{\infty j}^{*}$ is a base for the limit. This union is a subbase for any limit. But directedness implies that it is in fact a base.

Received by the editors May 17, 1998 and, in revised form, October 30, 2000.

2000 Mathematics Subject Classification. Primary 18B30, 54B30, 54D30, 54D45.

Key words and phrases. Directed inverse limits, spatial locales, locally compact spaces, locally compact locales, compact locales.

The first author was supported by a grant of the NSF of China.

(C)2002 American Mathematical Society 
We will also use the following theorem from [1] (see [9] or [10] for a choice-free proof).

Theorem 1. Directed inverse limits of compact locales are compact.

For terminology which is not explained here we refer the reader to [5].

\section{The COUnTEREXAMPle}

First we recall the notion of largest pointless sublocale introduced in [1], and described in more detail in 4. Not all locales have largest pointless sublocales but locales induced by sober $\mathbf{T}_{1}$ spaces do. If the largest pointless sublocale of a space $X$ exists, then it will be denoted by $p l(X)$. It can be computed either as the join of all pointless sublocales of $X$ or (if all points are complemented, i.e. locally closed) as the meet of all point complements. (Of course, meet and join refer to the lattice of all sublocales.) If $X \neq \emptyset$ is dense-in-itself, then each subspace $X \backslash\{x\}$ is dense in $X(x \in X)$. So the smallest dense sublocale is pointless (and nonzero), and therefore $p l(X)$ is nonzero.

Let $X$ be any dense-in-itself sober $\mathbf{T}_{1}$ space. Let $c X$ be the result of adding a closed point $*$ whose open neighborhoods are complements (in $c X$ ) of finite subsets of $X$. The space $c X$ is again sober (it is obtained by Artin glueing from $X$ and $\{*\}$ by the fringe map $j: \Omega(X) \rightarrow \Omega(\{*\})=2$ which satisfies $j(U)=1$ iff $U$ is cofinite) and compact $(c X-U$ is finite for any open neighborhood of $*$ ).

Consider the inverse system $\mathcal{X}$ consisting of spaces $X_{F}=c X \backslash\{x \mid x \in F\}$ where $F$ ranges over all finite subsets of $X$ ordered by reverse inclusion and maps the obvious embeddings $i_{F G}: X_{F} \rightarrow X_{G}(G \subseteq F)$. Its limit $X_{\infty}=\bigwedge_{F} X_{F}$ contains exactly one point $\infty$. The point $\infty$ is closed because it is the inverse image of $*$. Furthermore the only neighborhood of $\infty$ is $X_{\infty}$ because all open neighborhoods of $*$ in $c X$ get identified with some $X_{F}$ along the way. The open complement of $\infty$ is the intersection of all complements of $*$ in the $X_{F}$, i.e. it is the localic intersection of all subspaces $X-F$ in $X$, hence it is the largest pointless part $p l(X)$ of $X$. Since $X$ is dense-in-itself, $p l(X)$ is nonzero. So $X_{\infty}$ is not spatial.

Since $c X$ and all cofinite subspaces are locally compact if $X$ is compact Hausdorff and all inclusions are open we have proved the following proposition:

Proposition 1. There exist inverse systems consisting of locally compact locales and open maps whose inverse limit is not spatial.

Remark. In the statement of Proposition 1 "open map" can be replaced by "surjective local homeomorphism" because we can define another directed inverse system $\mathcal{Y}=\left\{Y_{F}, j_{F F^{\prime}}\right\}$ as follows: put $Y_{F}=\coprod\left\{X_{G} \mid G \subseteq F\right\}$ and for $F, F^{\prime} \subseteq X$ let $j_{F F^{\prime}}: Y_{F} \rightarrow Y_{F^{\prime}}$ be the map induced by the inclusions $X_{G} \rightarrow X_{G \cap F^{\prime}}$. That the limit of $\mathcal{Y}$ is not spatial follows immediately from the corresponding result for $\mathcal{X}$.

\section{A SUFFICIENT CONDITION FOR SPATIALITY}

Recall that a space $X$ is locally compact if each point $x \in X$ has the property that every open neighborhood of $x$ contains a compact neighborhood of $x$. Local compactness for locales is defined in terms of the way-below relation "«". Recall that for open sublocales $U, V$ of $X, V$ is said to be way below $U(V \ll U)$ iff every open cover of $U$ has a finite subset which covers $V$. A locale is said to be locally compact iff each open set is the join of opens way below itself. Note that it suffices to require that each open from a given basis is the join of opens way below itself. 
The two notions of local compactness are related as follows [5]:

(i) locally compact locales are spatial and the corresponding space is a locally compact sober space,

(ii) the locale induced by the topology of a locally compact space is a locally compact locale.

So there is a 1-1 correspondence between locally compact locales and locally compact sober spaces. For these the way-below relation can also be described as follows: $U \ll V$ iff there exists a compact sober subspace $K$ of $X$ such that $U \subseteq K \subseteq V$ iff there exists a compact sublocale $K$ of $X$ such that $U \leq K \leq V$.

In order to prove local compactness of the limit $L$ of an inverse system of locally compact locales it seems natural to look for conditions under which the way-below relation on $\Omega(L)$ is induced by the way-below relation on the $\Omega\left(X_{i}\right)$. Therefore we will only consider continuous maps $f: X \rightarrow Y$ between locally compact locales which preserve the way-below relation, i.e. if $U, V \in \Omega(Y)$ and $U \ll V$, then $f^{*}(U) \ll f^{*}(V)$. One way to ensure preservation of the way-below relation is to require that compactness of sober subspaces (respectively sublocales) is preserved under pullback, because for sober locally compact spaces (respectively locales) the way-below relation is equivalent to the existence of a sober compact subspace (respectively sublocale) between any two opens. So we get two notions. Say $f: X \rightarrow Y$ satisfies $\left(\mathrm{IC}_{s o b}\right)$ if pullbacks of compact sober subspaces of $Y$ along $f$ in the category of sober spaces are again compact, and it satisfies $\left(\mathrm{IC}_{l o c}\right)$ if the analogous condition holds in the category of locales. $\left(\mathrm{IC}_{l o c}\right)$ implies $\left(\mathrm{IC}_{s o b}\right)$ because spatial parts of compact locales are again compact 10. (If $f: X \rightarrow Y$ satisfies $\left(\mathrm{IC}_{l o c}\right)$ and $K \subseteq Y$ is a compact sober subspace, then $f^{-1}(K)$ is a compact sublocale of $X$, and hence its spatial part is a (sober and) compact subspace of $X$. But the latter is just the pullback of $K$ along $f$ in the category of sober spaces.) We believe that $\left(\mathrm{IC}_{s o b}\right)$ is strictly weaker than $\left(\mathrm{IC}_{l o c}\right)$ but we have no separating example.

The main result of this section is now the following:

Proposition 2. Let $\left\{X_{i}, f_{i j}: X_{i} \rightarrow X_{j} \mid i, j \in I, i \leq j\right\}$ be a directed inverse system of locally compact locales. If each map $f_{i j}$ satisfies $\left(\mathrm{IC}_{\text {sob }}\right)$, then the inverse limit $X_{\infty}$ is locally compact and hence spatial.

Proof. We have already noted that $\left(\mathrm{IC}_{s o b}\right)$ implies preservation of the way-below relation. We want to show that not only the maps $f_{i j}^{*}$ but also the maps $f_{\infty i}^{*}$ preserve the way-below relation, i.e. that for any $j \in I$ and for any $U \ll V$ in $\Omega\left(X_{j}\right)$ we have $f_{\infty j}^{-1}(U) \ll f_{\infty j}^{-1}(V)$. Given $U$ and $V$ as above we will construct a compact sublocale $K$ of $X_{\infty}$ such that $f_{\infty j}^{-1}(U) \leq K \leq f_{\infty j}^{-1}(V)$. Since $U \ll V$ in $\Omega\left(X_{j}\right)$ there exists a compact sober subspace $K_{j}$ of $X_{j}$ such that $U \subseteq K_{j} \subseteq V$. For each $i \leq j$ let $K_{i}=f_{i j}^{-1}\left(K_{j}\right)$ (again the spatial inverse image). Each $K_{i}$ is compact. Let $K$ be the inverse limit of $\left\{K_{i} \mid i \leq j\right\}$. Then $K$ is a compact sublocale of $X_{\infty}$ which satisfies $f_{\infty j}^{-1}(U) \leq K \leq f_{\infty j}^{-1}(V)$. So $f_{\infty j}^{-1}(U) \ll f_{\infty j}^{-1}(V)$.

But this implies that each open of the form $f_{\infty i}^{*}(U)$ for some $U \in \Omega\left(X_{j}\right)$ is the join of opens way-below it. But since these opens form a basis for $X_{\infty}$ this is all we need to conclude that the limit is locally compact.

Since finite products of locally compact locales are locally compact [5, 7] and hence spatial, we have the following corollary:

Corollary 1. Arbitrary products of compact locally compact locales are spatial. 
Remark. We don't know whether either of the implications

$$
\left(\mathrm{IC}_{l o c}\right) \Rightarrow\left(\mathrm{IC}_{s o b}\right) \Rightarrow f^{*} \text { preserves "«" }
$$

can be reversed in the realm of locally compact locales. Of course all three are weaker than properness [9]; they are strictly weaker because embeddings are proper iff they are closed, while inclusions of points (whether they are closed or not) always satisfy $\left(\mathrm{IC}_{l o c}\right)$. For Hausdorff locales the situation is less complicated. There the first implication can be reversed becaused then compactness implies spatiality. In this case, both $\left(\mathrm{IC}_{l o c}\right)$ and $\left(\mathrm{IC}_{s o b}\right)$ are also equivalent to properness, since $f$ can be extended to a map between the one-point-compactifications of its domain and codomain.

\section{REFERENCES}

[1] J. Isbell, Atomless parts of spaces, Math. Scand. 31 (1972), 5-32. MR 50:11184

[2] J. Isbell, Product spaces in locales, Proc. Amer. Math. Soc. 81 (1981), 116-118. MR 82c:54006

[3] J. Isbell, Direct limits of meet-continuous lattices, J. Pure App. Algebra 23 (1982), 33-35. MR 83a: 18015

[4] J. Isbell, First steps in descriptive theory of locales, Trans. Amer. Math. Soc. 327 (1991), 353-371. MR 92b:54078

[5] P.T. Johnstone, Stone Spaces, Cambridge University Press 1982. MR 85f:54002

[6] A. Joyal and M. Tierney, An extension of the Galois theory of Grothendieck, Mem. Amer. Math. Soc. No. 309, vol. 51, 1984. MR 86d:18002

[7] T. Plewe, Localic products of spaces, Proc. London Math. Soc. 73 (1996), 642-678. MR 97d:54012

[8] T. Plewe, Countable products of absolute C-delta spaces, Topology Appl. 74 (1996), 39-50. MR 97i:54015

[9] J. J. C. Vermeulen, Proper maps of locales, J. Pure App. Algebra 92 (1994), 79-107. MR 95d:54009

[10] He Wei and Liu Ying Ming, Steenrod's theorem for locales, Math. Proc. Cambridge Philos. Soc. (1998), 124, 305-307. MR 99c:18001

Department of Mathematics, Shaan Xi Normal University, Xi'an 710062, People's Republic of China

E-mail address: weihe@snnu.edu.cn

Current address: Department of Mathematics, Nanjing Normal University, Nanjing 210097, People's Republic of China

E-mail address: weihe@njnu.edu.cn

Department of Science and Engineering, Ritsumeikan University, Noji Higashi 1-1-1, Kusatsu-Shi, Shiga 525, JaPAN

E-mail address: till@theory.cs.ritsumei.ac.jp 